\title{
Retweet Prediction Based on Multidimensional Features
}

\author{
Xiaomeng Fu, Suyan Cheng, Li Zhao, and Jiaguo Lv \\ College of Medical Information and Engineering, Jining Medical University, Rizhao 276826, Shandong, China \\ Correspondence should be addressed to Jiaguo Lv; jglv2018@mail.jnmc.edu.cn
}

Received 10 November 2021; Revised 7 December 2021; Accepted 21 January 2022; Published 16 February 2022

Academic Editor: Mohamed Elhoseny

Copyright $\odot 2022$ Xiaomeng Fu et al. This is an open access article distributed under the Creative Commons Attribution License, which permits unrestricted use, distribution, and reproduction in any medium, provided the original work is properly cited.

With the wide use of artificial intelligence-driven mobile devices, more and more Chinese people take part in the Twitter-like social sites, which makes Weibo an excellent communication platform. In view of the wide application of information diffusion in various fields, Weibo has become one of the most important research issues in mobile social computing. In Weibo, the retweet statuses of tweets of other users are considered to be the key mechanism for spreading information. How to predict whether a tweet will be retweeted by a user has received increasing attention in recent years. Research shows that the users' retweet behavior is driven by their interest and personality. However, most previous works ignore the roles of users' personality in their retweet behavior. To this end, a prediction model MDF-RP (multidimensional feature-based retweeting prediction) including personality feature is proposed. The prediction model integrates the features from three dimensions, such as author, tweet, and user. And the personality score is obtained based on the well-known Big Five personality trait model. The experimental results under different classifiers show that the performances of MDF-RP features outperform the basic features. And the experiments of cross-validation also demonstrate the stability of MDF-RP features.

\section{Introduction}

In recent years, along with the extensive use of artificial intelligence-driven mobile devices, various mobile applications become more and more popular and profoundly change people's life. Due to the thousands of millions of users' participation, the mobile social network has been the most popular one. As the most popular online social media in China, Weibo has become an import platform for people to get and share information. In the Twitter-like social media sites, a user may share his or her life, works, and opinions with other users in the form of short message. A user may follow another user without his consent, and information can spread belong the following relationship among users, which forms an information diffusion network. Some characteristics of Weibo, such as the usage of “@, \#, ...” and weak friendship of users, make it different from other social media in terms of universality and immediacy of user participation, information diffusion mode, and speed. When users find interesting tweets and want to share them with their followers, they can retweet them using a button or other mechanisms to copy and post these tweets. The retweet function provided by this platform is usually considered to be the key mechanism for spreading information among users. To capture the dynamics of information diffusion occurring in social networks, it is necessary to model and predict user's retweet behaviors. Retweet prediction can predict whether a tweet can be retweeted by an individual and predict the diffusion scale of a given tweet. With the help of retweet prediction, some tweets that may trigger a largescale outbreak will be found as soon as possible. Retweet prediction plays an important role in hot-topic detection [1], personalized tweet recommendation [2,3], and information propagation prediction [4-6].

The rest of this article is organized as follows. The related work is described in Section 2. In Section 3, the prediction problem is formally defined. In Section 4, the MDF-RP method is introduced. Section 5 shows the experimental setup and evaluation results. Finally, the work is concluded in Section 6.

\section{Related Work}

Due to the enormous usefulness of prediction, a variety of studies have been conducted on the retweet prediction problem. The studies are carried out in two ways: one is 
author-centered, and the other is user-centered. For a given tweet, the former is employed to predict the scale of its retweet volume, and the latter is to predict whether a given user will retweet it or not. The author-centered work usually gets the influential factors via the statistics of the author, Twitter content, and post time. For example, those tweets with hashtag, URL, and other information are more likely to be retweeted. The more authoritative the author is, the more likely his tweet will be retweeted. Although the author-centered work has achieved good performance, it ignores the individual difference of each user. Therefore, researchers turned to the user-centered work. Different from the authorcentered work, in the user-centered prediction, besides the tweet and author features, user features should be taken into account. Under the MRF (Markov random field) energy optimization framework, Wang et al. [7] analyzed the effects caused by user attributes, tweet content, the constraints between users' retweet behavior, and the group retweet priors. Then, they constructed the corresponding energy function on the basis of the LR (logistic regression) model to predict users' retweet behaviors globally, which improved the accuracy of the proposed method. Taking into account the effects of tweet emotional difference on retweet prediction, Tang et al. [8] extended the semantic features of tweet text by Wikipedia knowledge base to mine users' interest and verified the effectiveness of the method with experiments. Analyzing the effects of users' interest, tweet contents, and others on retweet prediction, based on the factor graph model, Yang et al. [9] proposed a semisupervised framework to predict users' retweet behavior and found that the proposed method performed better than other existing methods. From the perspectives of users' dynamic interest, social relationship, and retweet behavior, the authors [10] proposed a prediction method combining multiple retweet features and found that social relationship had the greatest promotion on prediction accuracy. Wang et al. [11] extracted features from user profiles, user history, target tweet, and user network by a deep learning method to address the retweet prediction problem, which can effectively predict the retweet behavior of users in the experiments.

The existing author-centered work predicated users' retweet behaviors with their interest, which was mined from their historical tweets [9, 10, 12-16]. In psychology, psychologists attributed individuals' different reactions in the same environment to their personality. Personality is a stable mode of thoughts, emotion, and behaviors across time and situation [15]. Research showed that people's behavior in the social network was highly consistent with that in reality. Users' retweet behavior in Weibo was an explicit reflection of their personality. Tweet content was reflective of the author's personality. In the personality theory, Big Five personality [17] is the most effective one in evaluating individual differences. Big Five personality describes an individual's personality traits from five dimensions: extroversion (EXT), neuroticism (NEU), agreeableness (AGR), conscientiousness (CON), and openness (OPN). Therefore, the personality trait score obtained from a user's tweets will be selected as an import feature for retweet prediction problem.
In conclusion, taking the user as the center, considering the factors affecting a user's retweet behavior from three dimensions of author, tweet text, and user, the multidimensional features for retweet prediction were obtained. These features were fused together in a classic decision tree algorithm, which improved the prediction performance greatly.

\section{Problem Definition}

Weibo network can be modeled as a weighted directed graph $G(V, E, W)$, where $V, E$, and $W$ denote the node set, edge set, and edge weight set, respectively. In $G, u$ and $v$ are two users in the Weibo network. If $u$ is a follower of $v$, then there is an edge $e(u, v)$ in graph, and $w(u, v)$ is the weight of edge $e(u$, $v)$. In fact, $w(u, v)$ is usually estimated by the interaction behaviors between node $u$ and $v$.

Let $m$ be a tweet posted by $v$, and $H(u, v, m)=\left(f_{1}, f_{2}, f_{3}\right.$, ...) is a vector composed of features that can influence the retweet behavior of $u . p(r=1 \mid H(u, v, m))$ represents the probability that $u$ retweets $m$, where $r$ is a binary variable indicating whether $u$ retweets $m$. Given $u, v$, and $m$, the retweet prediction problem is how to determine the vector $H$ $(u, v, m)$ and then estimate the retweet probability $\mathrm{p}(r=1 \mid H$ $(u, v, m))$.

This work focuses on the analysis of the factors influencing users' retweet behavior. The author-centered work usually obtains the prediction features from the statistics of the hot tweets. However, the features of hot tweets only reflect the overall trend of all users' retweet behavior and ignore the individual difference of each user. So, the user features are taken into account in this work.

The working procedure of algorithm MDF-RP is shown in Figure 1. As can be seen from Figure 1, the algorithm first extracts the features of the author, tweet, and user from the original dataset and then integrates these features with some classifier to predict whether the tweet will be retweeted by the user.

\section{Proposed Method}

The selection of retweet features is crucial to the performance of the prediction method. In this work, the prediction features are extracted from the information, which comes from user $u$, author $v$, tweet $m$, and the historical tweet sets of $u$ and $v$.

4.1. Author Features. On the basis of the previous work, some author features, such as the number of fans, whether the author is authenticated, average number of tweets posted by the author every day and the popularity are chosen to predicate the retweet behavior.

4.1.1. Number of Fans. The number of fans is very important to evaluate the influence of a tweet author. The feature reflects the size of the author's audience. The statistics on the real dataset show that the number of fans of a tweet author is 


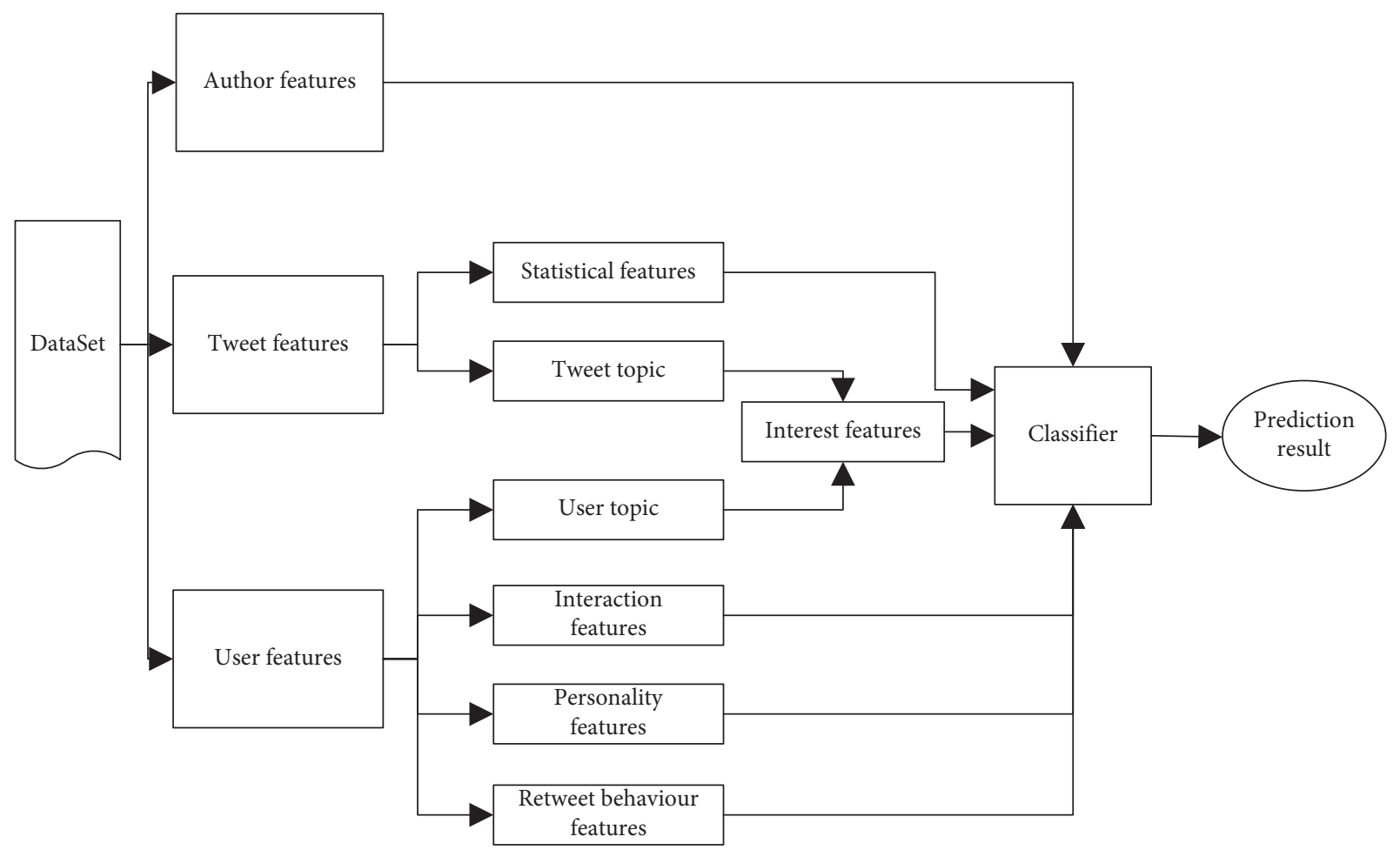

FIGURE 1: Working procedure of algorithm MDF-RP.

positively correlated with the number of times his tweets are retweeted.

4.1.2. Authenticated or Not. Authenticated user is the user whose profile has been authenticated by the Weibo platform. It is obvious that an authenticated user has higher credibility than an ordinary one. The statistics on the real dataset also show that the number of retweets posted by authenticated users is 3.2 times that of unauthenticated users.

4.1.3. Average Number of Tweets Posted Daily. The number of tweets posted daily means the activity of the author. A tweet author needs to post more tweets to maintain and increase his fans. Generally speaking, for a tweet author, the more fans he has, the more likely his post is to be retweeted. The average number of tweets daily of $v$ is calculated as follows:

$$
\operatorname{daily}(v)=\frac{\operatorname{posts}(v, t)}{\operatorname{days}(t)}
$$

where posts $(v, t)$ and days $(t)$ refer to the number of tweets posted by $v$ and the number of days during time $t$.

4.1.4. Popularity. To a certain extent, popularity reflects the approval degree of an author's posts. Suppose $n(m)$ denotes the retweet number of post $m$. Given a threshold $\zeta$, if $n(m)$ is greater than $\zeta, m$ is popular. As for a tweet author, popularity refers to the proportion of the popular posts among all his posts. For a tweet author $v$, the popularity is calculated as follows:

$$
\operatorname{popularity}(v)=\frac{|\operatorname{hotposts}(v)|}{|\operatorname{posts}(v)|},
$$

where hotposts $(v)$ is the tweet set of popular posts of $v$, and $\operatorname{posts}(v)$ refers to the tweet set of $v$.

4.2. Tweet Features. Tweet features refer to whether the tweet contains some special information found in popular tweets, such as Hashtag, URL, @, picture, video, and whether it is original. Statics show that when a tweet contains this special information, it is more likely to be retweeted. Tweet features employed in this work will be described in detail in part 5 .

4.3. User Features. Four kinds of user features, including retweet behavior preference, interest, personality, and social interactions, are taken into account to predict the retweet behavior.

4.3.1. Retweet Preference. In the Weibo platform, the user has a variety of interaction behaviors, such as retweet, comment, and like. Retweet preference features are used to describe whether a user tends to retweet a tweet. They are described in two ways: retweet behavior preference and retweet activity.

As for a Weibo user, retweet behavior preference is the tendency of his retweet behavior by comparison with other interaction behaviors. Statistics of the Weibo platform show that users tend to spend their limited time in their favorite interaction behaviors. Compared to other users, those with 
high retweet behavior preferences are more likely to retweet a post. The retweet behavior preference of a user is evaluated as follows:

$$
\operatorname{pre}(u)=\frac{w_{r} N_{r}(u)}{w_{r} N_{r}(u)+w_{c} N_{c}(u)+w_{l} N_{l}(u)},
$$

where $N_{r}(u), N_{c}(u)$, and $N_{l}(u)$ are the times of retweet, comment, and like of $u ; \mathrm{w}_{r}, \mathrm{w}_{c}$, and $\mathrm{w}_{\mathrm{l}}$ are the weights of these three actions, and $\mathrm{w}_{r}+\mathrm{w}_{c}+\mathrm{w}_{l}=1$. Generally speaking, retweet action requires more emotion, followed by comment and like actions. In experiments, the weight values are obtained empirically according to the average number of these three behaviors in our dataset. Suppose nr, nc, and nl are the average times of retweet, comment, and like in the dataset, $\mathrm{w}_{\mathrm{r}}: \mathrm{w}_{\mathrm{c}}: \mathrm{w}_{\mathrm{l}}=1 / n_{\mathrm{r}}: 1 / n_{\mathrm{c}}: 1 / n_{\mathrm{l}}$. Finally, $\mathrm{w}_{\mathrm{r}}, \mathrm{w}_{\mathrm{c}}$, and $\mathrm{w}_{\mathrm{l}}$ are set to $0.6,0.3$, and 0.1 , respectively.

Retweet activity reflects a user's activity in retweet action. Statistics show that only a few users have high retweet times, and the distribution of users' retweet times obeys long-tail distribution approximately. To avoid the impact of long-tail distribution on data standardization, grade classification is employed to estimate the retweet activity. First, all users are sorted by their retweet times ascending, and then, they are divided into $n$ levels. The number of users in each level is $[|v| / N]$. Let $N_{\mathrm{r}}(v)$ represent the retweet times of user $v$ and $k$ is the maximum retweet times in level $k\left(\eta_{0}=0\right)$. If $N_{\mathrm{r}}(v)$ is in $\left(\eta_{k-1}, \eta_{\mathrm{k}}\right]$, then $v$ is in level $k$. The retweet activity act $(v)$ is calculated as follows:

$$
\operatorname{act}(v)=\frac{k-1}{n}+\frac{N_{r}(v)}{n * \eta_{k}} .
$$

4.3.2. Retweet Interest Features. Psychological research shows that one's interest will not change in a short time. Users' retweet behavior in Weibo is driven by their interests. In Weibo, users' interest can be mined from those tweets posted or retweeted by themselves. Due to the diversity of users' interest, the tweet content can be divided into different topics with algorithm LDA, and then, interest feature will be extracted from those topics.

Let $D(u)$ denote all tweets of user $u$. Firstly, the tweets of $D(u)$ are clustered by algorithm LDA to obtain $n$ topics. Let $D(u, k)$ denote all tweets in $D(u)$ of the kth topic. For each topic of $D(u)$, top 10 words with the highest TF-IDF are taken as the feature words. With these feature words, the feature vector $i(u, k)(\mathrm{k} \in\{1,2,3, \ldots, n\})$ will be obtained, which is used to represent the kth interest of user $u$. The importance of $i(u, k)$ is calculated as follows:

$$
i(u, k)_{\text {weight }}=\frac{M(D(u, k))}{M(D(u))},
$$

where $M(D(u))$ and $M(D(u, k))$ denote the tweet number of $D(u)$ and $D(u, k)$, respectively. With the same method, the feature vector $j(m)$ of tweet $m$ can be obtained. As for user $u$, its retweet interest feature $\mathrm{fac}(u, m)$ for tweet $m$ is calculated as follows:

$$
\operatorname{fac}(u, m)=\sum_{k=1}^{n} i(u, k)_{\text {weight }} * \operatorname{sim}(i(u, k), j(m)),
$$

where $\operatorname{sim}(i(u, k), j(m))$ represents the similarity of vector $i(u, k)$ and $j(m)$. In this work, the similarity is calculated with cosine similarity, which is described as follows:

$$
\operatorname{sim}(i(u, k), j(m))=\frac{i(u, k) * j(m)}{\|i(u, k)\| \times\|j(m)\|} .
$$

4.3.3. Interaction Features. In social network, users' behavior is usually influenced by their friends. The influence is estimated by the number of retweets, comments, and likes of $u$ on author $v$ in a period of time (one month is selected in the experiments). Let $N_{\mathrm{r}}(v, u), N_{\mathrm{c}}(v, u)$, and $N_{\mathrm{l}}(v, u)$ denote the number of retweets, comments, and likes of $u$ on $v$, and then, the influence $\inf (v, u)$ is calculated as follows:

$$
\inf (v, u)=w_{r} N_{r}(v, u)+w_{c} N_{c}(v, u)+w_{l} N_{l}(v, u),
$$

where $\mathrm{w}_{\mathrm{r}}, \mathrm{w}_{\mathrm{c}}$, and $\mathrm{w}_{\mathrm{l}}$ denote the weight of retweet, comment, and like actions.

4.3.4. Personality Features. There are two kinds of methods to obtain users' personality from their text information: one is based on machine learning, and the other is based on psychological dictionary. As for the method based on machine learning, it is difficult to obtain the training set containing users' real personality. The acquisition of users' personality is generally carried out by questionnaire survey in psychology, which will consume a lot of human and material resources. The method based on psychological dictionary needs the quantitative relationship between the function of words and personality traits.

LIWC (Linguistic Inquiry and Word Count) [18] was released by Pennebaker et al., which discovered the relationship between personality traits and language habits by calculating the frequency of specific words. LIWC focuses on the analysis of English text. In order to facilitate the personality analysis of Chinese texts, with the official authorization of LIWC, Huang et al. [19] transformed it into C-LIWC and SC-LIWC, which are suitable for the analysis of traditional Chinese text and Simplified Chinese text, respectively.

In this work, SC-LIWC dictionary is employed to analyze the personality of tweet users, and the Big Five personality model is used as the psychological theory to study the personality of users, and the personality traits of users reflected in tweet texts are analyzed. When conducting personality analysis based on SC-LIWC, emotion words and degree adverbs are also necessary to be considered. Emotional words reflect the user's attitude and feelings towards the relevant object, which can be divided into a positive one and a negative one. Degree adverb expresses the degree of emotion. The UTNSD-Simplified Chinese Emotion Polarity Dictionary published by National Taiwan University divides 11086 emotion words into 2810 positive words and 8276 negative words.

HowNet is a common sense knowledge base developed by the Chinese Academy of Sciences. A dictionary for sentiment analysis (beta) was released by HowNet in 
October 2007. On the basis of the dictionary of LIWC2007 and C-LIWC, a Chinese language psychological analysis system TextMind was developed by the Institute of Psychology, Chinese Academy of Sciences. In this system, the relationship between 71 function words in SC-LIWC and each dimension of the Big Five personality was statistically quantified. In SC-LIWC, a word may have multiple functions. TextMind provides the corresponding table between function words and personality trait score. Based on this, the personality trait score of the tweet user can be calculated.

Definition 1 Tweet personality. In the Big Five model [17], personality is composed of five traits. The different proportion of these five traits in personality forms different personality performance. Personality is defined as follows:

Personality $=\left(S_{-}\right.$open, $S_{-}$cons, $S_{-}$extr, $S_{-}$agree, $S_{-}$neur $)$,

where S_open, S_cons, S_extr, S_agree, S_ Neur indicate the scores of personality trait in openness, conscientiousness, extroversion, agreeableness, and neuroticism, respectively.

Definition 2 BFM. BFM denotes the importance of word $\mathrm{w}$ in the ith dimension of the Big Five personality. It is calculated as follows:

$$
\operatorname{BFM}(w, i)=\frac{\sum_{j=1}^{n} p(j, i)}{n},
$$

where $n$ is the number of function words of word $\mathrm{w}$, and $p$ $(j, i)$ denotes the relationship between the $j$ th function word and the ith personality factor. In formula $(10), p(j, i)$ is obtained from TextMind.

BFM takes into account the relationship between the function words and the personality traits. However, the personality trait score is also related to the importance of the word in the tweet. In this work, TF-IDF is employed to denote the importance of word $\mathrm{w}$ in tweet $m$. PTS $(m, \mathrm{w}, i)$ denotes the $i$ th personality trait score of word $\mathrm{w}$ in tweet $m$, and it is estimated as follows:

$$
\operatorname{PTS}(m, w, i)=\operatorname{BFM}(w, i) * t f-\operatorname{idf}(w, m),
$$

where $\operatorname{tf}-\operatorname{idf}(w, m)$ denotes the tf-idf value of word $\mathrm{w}$ in tweet $m$.

When calculating the tweet personality score of tweet $m$, not only the personality score and word frequency of each word $\mathrm{w}$ in $m$, but also the emotional words and degree adverbs in front of $\mathrm{w}$ should be taken into account. The calculation algorithm of the tweet personality score is described in Algorithm 1.

\section{Experiments}

5.1. Datasets. In order to evaluate the method in this work, we crawled data from Weibo, which is a social network in China like Twitter. We randomly selected 80 users as the seed users from 8 fields such as technology, entertainment, and tourism and then crawled 44273 tweets posted or retweeted by these users from June 1, 2018 to July 31, 2018. Then, we obtained the followed list of these seed users including 3,352 users and got 316,829 tweets published by these users. The data in June are used as the historical data, and the rest data are employed as experimental data. The historical data are used to mine retweet habits, and the experimental data are used to train and test the prediction model.

In order to improve the credibility of the experiment, cross-validation is employed to test the performance of the retweet prediction method. In experiments, 4 -fold crossvalidation is used. Two groups of experiments are designed to verify the effectiveness and stability of the proposed method. One is used to verify the contribution of the proposed features to improve the performance of retweet prediction, and the other is to verify the stability of the proposed features on different classifiers and training sets.

5.2. Evaluation. In order to measure the performance of the retweet prediction method, precision, recall, and F1-score are used in this work. These measures are defined as follows:

$$
\begin{aligned}
\text { precision } & =\frac{\# \text { correctly classified as retweet }}{\# \text { classified as retweet }}, \\
\text { recall } & =\frac{\# \text { correctly classified as retweet }}{\text { \#true retweet }}, \\
F 1-\text { score } & =\frac{2 * \text { precision } * \text { recall }}{\text { precision }+ \text { recall }} .
\end{aligned}
$$

5.3. Experimental Results. The experiment environment of this work is as follows:

\section{Hardware: CPU: Intel(R) Xeon(R) CPU E5-2696 v2 @ $2.50 \mathrm{GHz}$; Memory: $48 \mathrm{G}$ \\ OS: CentOS 7}

On the basis of the features of the author, tweet, and user, classic classifiers including decision tree C4.5 and LR are employed to predict the individual retweet behavior.

5.3.1. Experimental Results. All the features employed in method MDF-RP are shown in Table 1.

In order to verify the effectiveness of the proposed method, author features and tweet features (features 1-9) are selected as benchmarks. The benchmark features and author features are employed in algorithm C4.5, and the experimental results are shown in Table 2.

As shown in Table 2, after user features are added, F1score is increased by $6.519 \%$. Comparing the basic features, user features fully consider the key factors such as users' retweet behavior preference, activity, interest in tweet, personality trait scores, and the influence from the tweet author, which effectively improves the performance of algorithm MDF-RP.

In order to obtain the contribution of each user feature to the performance improvement, comparative experiments on the prediction performance of each user feature are 


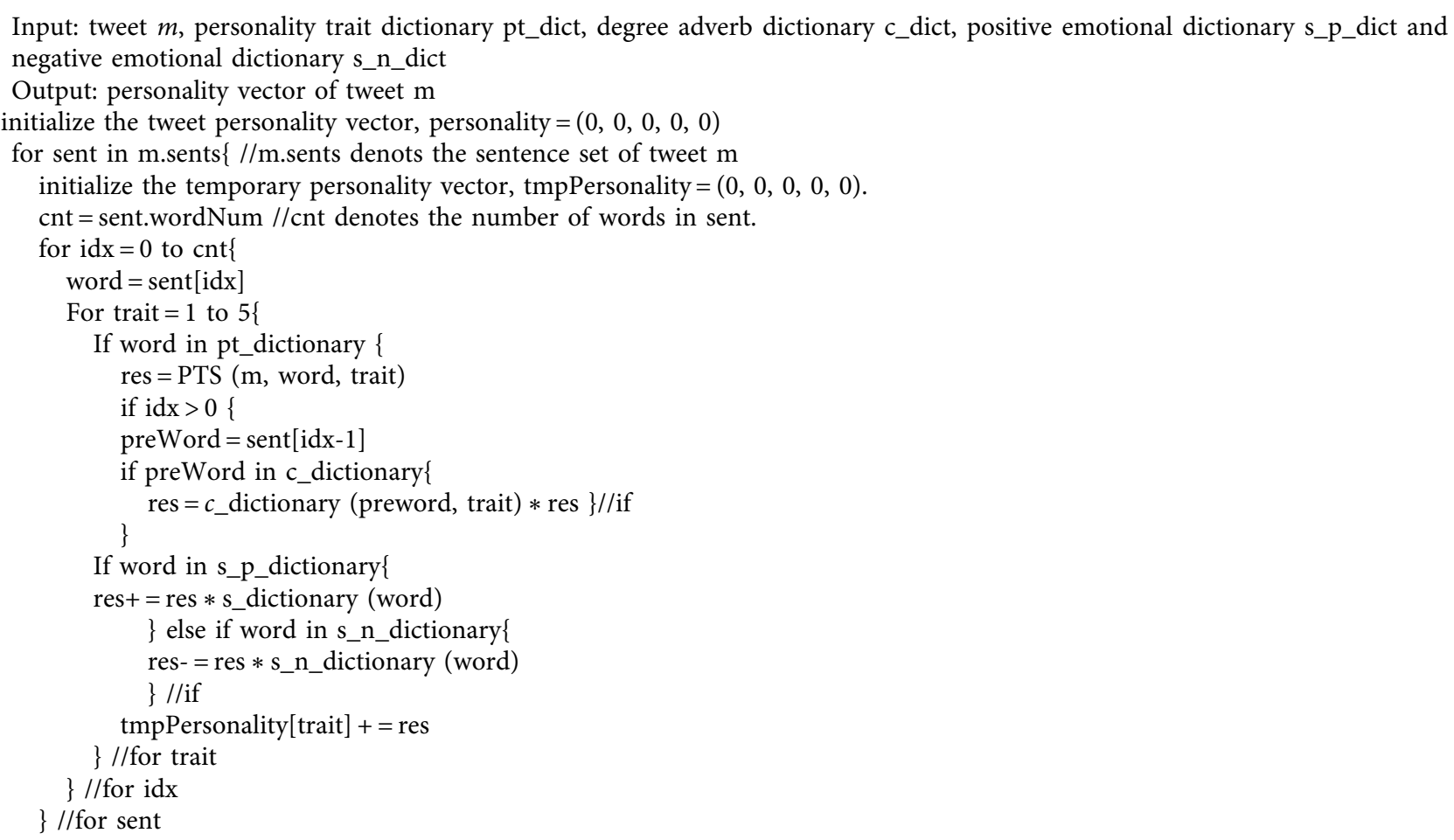

Algorithm 1: get_tweet_personality_score.

TABLE 1: Summary of features in MDF-RP.

\begin{tabular}{|c|c|c|}
\hline Category & Feature & Description \\
\hline \multirow{4}{*}{ Author features } & f_a_follows & Number of follows of the author \\
\hline & f_a_if_aut & Whether the author is authenticated \\
\hline & f_a_daily & The average number of tweets posted by the author daily \\
\hline & f_a_popularity & A feature denotes the popularity of an author's tweets \\
\hline \multirow{5}{*}{ Tweet features } & f_m_if_hashtag & Whether the tweet contains hashtag \# \\
\hline & f_m_if_url & Whether the tweet contains hyperlink \\
\hline & f_m_if_@ & Whether the tweet contains@ \\
\hline & f_m_if_multimedia & Whether the tweet contains some multimedia materials \\
\hline & f_m_if_org & Whether the tweet is original \\
\hline \multirow{5}{*}{ User features } & f_u_ret_pre & The retweet behavior preference of the user \\
\hline & f_u_act & The retweet activity of the user \\
\hline & f_u_int & The interest similarity of the user and the given tweet \\
\hline & F_u_inf & The influence of the author on the user \\
\hline & f_u_personality & The personality trait vector of the user \\
\hline
\end{tabular}

TABle 2: Performance of retweet prediction with benchmark methods and MDF-RP.

\begin{tabular}{lcccc}
\hline Feature & Precision & Recall & F1-score & $\begin{array}{c}\text { F_promotion_rate } \\
(\%)\end{array}$ \\
\hline Basic_Features & 0.793 & 0.742 & 0.767 & - \\
MDF-RP & 0.851 & 0.785 & 0.817 & 6.519 \\
\hline
\end{tabular}

conducted, and the experimental results are shown in Table 3.

It can be seen from Table 3 that the performance promotion of $\mathrm{f}$ _u_personality is the highest, reaching $6.519 \%$, and the promotion of $\mathrm{f}$ _u_ret_pre is the lowest, reaching $0.780 \%$.
5.3.2. Performances of Different Classifiers. In order to verify the performance of MDF-RP features, experiments of basic features and MDF-RP features under different classifiers are conducted. To verify the stability of these features, cross-validation is employed. K-Fold cross-validation divides the dataset into $k$ parts. It takes $k-1$ parts as the training set and the remaining part as the test set in turn. It estimates the prediction performance of the algorithm by the mean of $k$ experimental results. The experimental results of basic features and MDF-RP features under different classifiers are shown in Figures 2-4.

It can be seen from Figures 2-4 that under different algorithms F1-score of MDF-RP features is higher than that of the basic features. The MDF-RP features of all algorithms 
TABLE 3: Contribution of user features to retweet prediction.

\begin{tabular}{lcccc}
\hline Feature & Precision & Recall & F1-score & F_promotion_rate (\%) \\
\hline Basic_Features & 0.793 & 0.742 & 0.767 & - \\
Basic_Features +f_u_ret_pre & 0.801 & 0.747 & 0.773 & 0.780 \\
Basic_Features +f_u_act & 0.820 & 0.760 & 0.789 & 2.876 \\
Basic_Features +f_u_int & 0.824 & 0.762 & 0.792 & 3.234 \\
Basic_Features + F_u_inf & 0.824 & 0.748 & 0.784 & 2.252 \\
Basic_Features +f_u_personality & 0.828 & 0.765 & 0.795 & 3.699 \\
MDF-RP & 0.851 & 0.785 & 0.817 & 6.519 \\
\hline
\end{tabular}

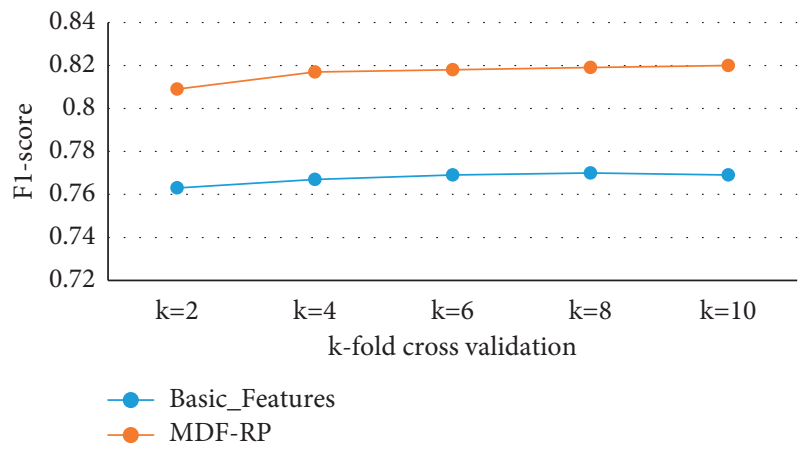

Figure 2: Prediction performance of algorithm C4.5.

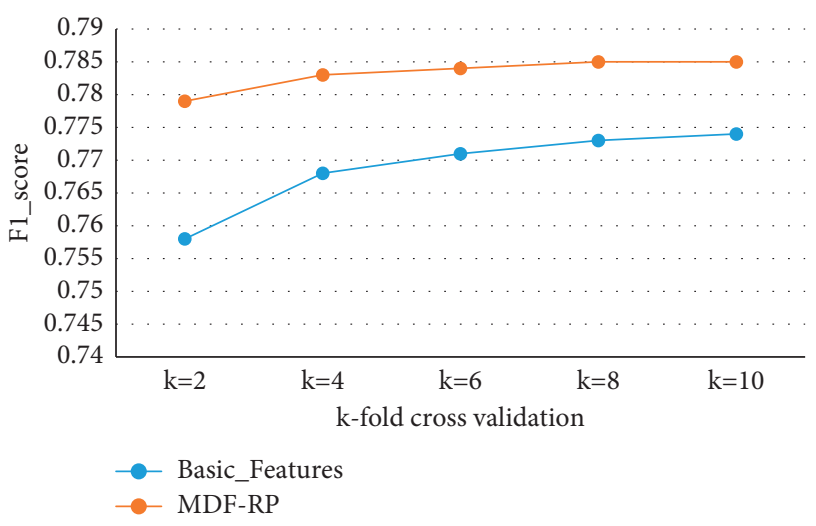

FIgURE 3: Prediction performance of algorithm logistic regression.

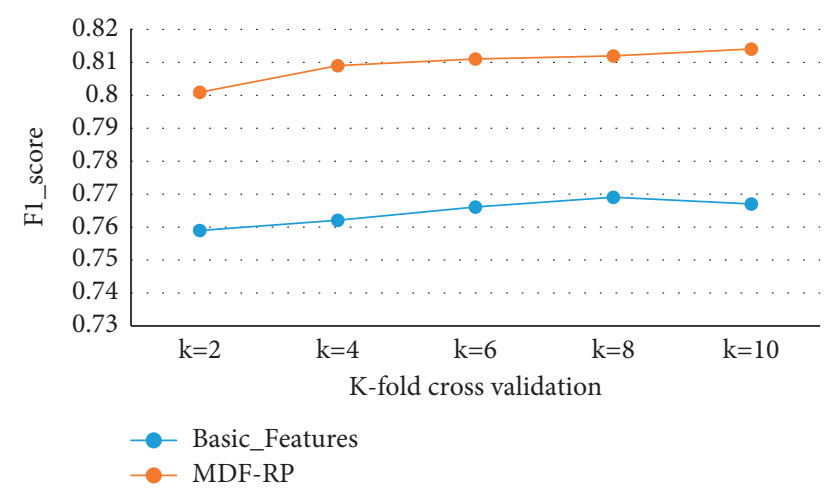

FIgURe 4: Prediction performance of algorithm SVM.

can obtain a high F1-score in the case of a small training set. In addition, F1-score of MDF-RP can be gradually improved with the rise of the training data size.
In algorithm C4.5, under 2-fold and 10-fold cross-validation, compared with the basic features, F1-scores of MDF-RP features are increased by $6.03 \%$ and $6.63 \%$, respectively. Under 2-fold cross-validation, the F1-score of MDF-RP features is 0.809 . From 2 -fold to 10 -fold crossvalidation, F1-scores of MDF-RP features increase by $1.36 \%$. However, the F1-score of basic features decreases under the 10 -fold cross-validation.

In algorithm LR, under 2-fold and 10-fold cross-validation, compared with the basic features, F1-scores of MDFRP features are increased by $2.77 \%$ and $1.42 \%$, respectively. Under 2-fold cross-validation, the F1-score of MDF-RP features is 0.779 . From 2 -fold to 10 -fold, the F1-score of MDF-RP is improved by $0.77 \%$, whereas the F1-score of basic features is improved by $2.11 \%$.

In algorithm SVM, under 2-fold and 10-fold crossvalidation, compared with the basic features, F1-scores of MDF-RP features are increased by $5.24 \%$ and $5.77 \%$, respectively. Under 2-fold cross-validation, the F1-score of MDF-RP features is 0.801 . From 2 -fold to 10 -fold, the F1score of MDF-RP is improved by $1.04 \%$, whereas the F1score of basic features is improved by $1.59 \%$.

The experimental results under different classifiers show that, compared with the basic features, the user features can effectively predict the user's retweet behavior for a given tweet. In experiments, the classifier with MDF-RP features can obtain high performance on a smaller training set. Moreover, with the increase in the size of the training data, the performance improves steadily. This is because the MDF-RP features contain the information of tweet content, user interests, user personality, user interaction, and others, which can describe the features of user's retweet behavior. So, the classifier with these features trained on a small dataset can obtain better and more stable performance.

\section{Conclusion}

With regard to the retweet prediction problem of a given user, from the dimensions of the author, tweet content, and user, a prediction method based on multidimensional features is proposed in this work.

(1) In the dimension of the tweet author, from the perspectives of the influence, credibility, activity, and the quality of his tweets, four features are designed, including number of fans, authenticated user or not, number of tweets posted daily, and popularity, which effectively measure the influence of the tweet author on whether the tweet will be retweeted 
(2) In the dimension of the tweet content, according to the common features of those hot posts, five features are designed, including whether the tweet contains topic tags, hyperlinks, @, multimedia materials, and whether it is original, which measure the impact of the tweet's statistical feature effectively

(3) In the dimension of the tweet user, five features based on his personality, retweet behavior preference, retweet activity, interest in the tweet content, and the influence from the tweet author are chosen to effectively measure the impact on the retweet prediction problem

Based on these multidimensional features, a tweet prediction method is proposed. The effects of these multidimensional features on different classification methods are verified in the real datasets. The experiments show that these features effectively improve the prediction performance. The prediction performance of algorithm C4.5 is the highest, and it can achieve the best prediction effect on a smaller training set. In fact, users usually play different roles in the dissemination of tweets with different themes. As future work, we plan to extend this work by incorporating user's role in tweet propagation to further improve the performance of retweet prediction.

\section{Data Availability}

The data that support the findings of this study are available from the corresponding author upon reasonable request.

\section{Conflicts of Interest}

The authors declare no potential conflicts of interest with respect to the research, authorship, and/or publication of this article.

\section{Acknowledgments}

This work was partially supported by the National Innovation Training Foundation for college students (Grant no. 201910443011) and Supporting Fund for Teachers' Research of Jining Medical University (Grant no. JYFC2019KJ038).

\section{References}

[1] Y. Wang, Z. Zhang, C. Chang, and M. A. Zia, "Identifying and tracking topic-level influencers in the microblog streams," Machine Learning, vol. 107, no. 3, pp. 551-578, 2018.

[2] C. De Maio, G. Fenza, M. Gallo, V. Loia, and M. Parente, "Time-aware adaptive tweets ranking through deep learning," Future Generation Computer Systems, vol. 93, no. 924-932, 2019.

[3] J.-H. Zhao, D.-L. Zeng, J.-T. Qin, H.-M. Si, and X.-F. Liu, "Simulation and modeling of microblog-based spread of public opinions on emergencies," Neural Computing and Applications, vol. 33, no. 2, pp. 547-564, 2021.

[4] Z.-Y. Han, L.-L. Kong, and H.-L. Qi, “Time segment language model for microblog retrieval," Neural Computing and Applications, vol. 33, no. 10, pp. 4763-4777, 2021.
[5] W. Liu, M. He, L. H. Wang, Y. Liu, H. W. Shen, and X. Q. Cheng, "Research on microblog retweeting prediction based on user behavior features," Chinese Journal of Computers, vol. 39, no. 10, pp. 1992-2006, 2016.

[6] B. Jiang, J. Liang, Y. Sha, and L. H. Wang, "Message clustering based matrix factorization model for retweeting behavior prediction," in Proceedings of the 24th ACM International on Conference on Information and Knowledge Management, pp. 1843-1846, Melbourne, Australia, 2015.

[7] W. Wang, X. W. Zhang, G. H. Ren, D. X. Qin, and L. L. Liu, "Predicting microblog user retweet behaviors on energy optimization," Acta Electtronica Sinica, vol. 45, no. 12, pp. 2987-2996, 2017.

[8] X. B. Tang and Y. L. Luo, "Integrating emotional divergence and user interests into the prediction of microblog retweeting," Library and Information Service, vol. 61, no. 9, pp. 102-110, 2017.

[9] Z. Yang, J. Guo, K. Cai, J. Tang, J. Z. Li, and L. Zhang, "Understanding retweeting behaviors in social networks," in Proceedings of the 19th ACM International Conference on Information and Know Ledge M Anagement, pp. 1633-1636, ACM, Toronto, Canada, 2010.

[10] B. Bi and J. Cho, "Modeling a retweet network via an adaptive Bayesian approach," in Proceedings of the 25th International Conference on World Wide Web, pp. 459-469, Montreal, Canada, 2016.

[11] C. Wang, Y. Fan, Y. Du, and Z. Sun, "Predict individual retweet behavior based on multi-feature," IOP Conference Series: Materials Science and Engineering, vol. 790, no. 1, Article ID 012046, 2020.

[12] S. N. Firdaus, C. Ding, and A. Sadeghian, "Retweet prediction considering user's difference as an author and retweeter," in Proceedings of the 2016 IEEE/ACM International Conference on Advances in Social Networks Analysis and Mining (ASONAM), pp. 852-859, IEEE, San Francisco, CA, USA, 2016.

[13] Q. Zhang, Y. Gong, Y. Guo, and X. J. Huang, "Retweet behavior prediction using hierarchical dirichlet process," Proceedings of the AAAI Conference on Artificial Intelligence, vol. 29, no. 1, 2015.

[14] Q. Zhang, Y. Gong, and J. Wu, "Retweet prediction with attention-based deep neural network," in Proceedings of the 25th ACM International on Conference on Information and Knowledge Management, pp. 75-84, Indianapolis, IN, USA, 2016.

[15] D. C. Funder, "On the accuracy of personality judgment: a realistic approach," Psychological Review, vol. 102, no. 4, pp. 652-670, 1995.

[16] Z. M. Xu, D. Li, T. Liu, S. Li, G. Wang, and S. L. Yuan, "Measuring similarity between microblog users and its application," Chinese Journal of Computers, vol. 37, no. 1, pp. 207-218, 2014.

[17] L. R. Goldberg, J. A. Johnson, H. W. Eber et al., "The international personality item pool and the future of publicdomain personality measures," Journal of Research in Personality, vol. 40, no. 1, pp. 84-96, 2006.

[18] J. W. Pennebaker, "Linguistic styles: language use as an individual difference," Journal of Personality \& Social Psychology, vol. 77, no. 6, 1999.

[19] C. L. Huang, C. K. Chung, and N. Hui, "The development of the Chinese linguistic inquiry and word count dictionary," Chinese Journal of Psychology, vol. 54, no. 2, pp. 185-201, 2012. 